\title{
A novel mast cell-dependent allergic peritonitis model
}

\author{
Hadas Pahima ${ }^{1}$, Pier Giorgio Puzzovio ${ }^{1}$, and Francesca Levi-Schaffer ${ }^{1}$ \\ ${ }^{1}$ Hebrew University of Jerusalem Faculty of Medicine
}

January 20, 2021

\begin{abstract}
Background: Typical murine models of allergic inflammation are induced by the combination of ovalbumin and aluminum hydroxide. However, accumulating evidence indicates that, in models of asthma and atopic dermatitis, allergic inflammation can be generated in the absence of aluminum hydroxide. Moreover, co-administration of S. aureus enterotoxin B with ovalbumin can enhance inflammation. Objective: The objective of this study was to establish a rapid and mast cell-dependent murine model of allergic inflammation by inducing allergic peritonitis using ovalbumin and S. aureus enterotoxin B. Methods: Allergic peritonitis was induced in C57BL/6 mice by subcutaneous sensitization and intraperitoneal challenge with ovalbumin and S. aureus enterotoxin B. Disease characteristics were assessed by flow cytometry, ELISA, Trypan Blue exclusion and colorimetric assays. Results: Time course of the allergic peritonitis revealed a peak of peritoneal inflammation $48 \mathrm{~h}$ after challenge, as assessed by total cells and eosinophil counts. Decrease of cell numbers started $96 \mathrm{~h}$ post challenge with complete clearance within 168h. Moreover, significantly higher levels of tryptase and increased vascular permeability were found 30 min following challenge. Allergic inflammation induction by ovalbumin and S. aureus enterotoxin B was impaired in mast cells deficient mice and partially restored by mice reconstitution with bone marrow derived mast cells, indicating the mast cell role in this model. Conclusion: We present a novel model of allergic peritonitis that is mast cell-dependent, simple and robust. Moreover, the use of S. aureus enterotoxin B better resembles human allergic inflammation, which is known to be characterized by the colonization of Staphylococcus aureus.
\end{abstract}

\section{A novel mast cell-dependent allergic peritonitis model}

Running title: OVA/SEB allergic peritonitis

Hadas Pahima $^{1}$, Pier Giorgio Puzzovio ${ }^{1}$ and Francesca Levi-Schaffer ${ }^{1}$

1 Pharmacology and Experimental Therapeutics Unit, School of Pharmacy, Institute for Drug Research, Faculty of Medicine, The Hebrew University of Jerusalem, Jerusalem, Israel.

*Corresponding Author: Francesca Levi-Schaffer, PharmD, PhD, Pharmacology \& Experimental Therapeutics Unit, Institute for Drug Research, School of Pharmacy, Faculty of Medicine, POB 12271, The Hebrew University of Jerusalem, Jerusalem 91120, Israel, Tel: 972-2-6757512, Fax: 972-2-6758144, E-mail: francescal@ekmd.huji.ac.il

Keywords: Allergic Inflammation; Allergic Peritonitis; Animal Models; Eosinophils; Mast Cells

\section{Abbreviations}

AD-atopic dermatitis

AEU-Allergic Effector Unit

AI-allergic inflammation

Alum-Aluminum hydroxyde 
AP-allergic peritonitis

AR-allergic rhinitis

BMMCs- Bone Marrow-derived Mast Cells

DCs-dendritic cells

Eos- Eosinophils

FC-flow cytometry

KO-knockout

i.p.- intraperitoneal

i.v.-intravenous

MCs- Mast Cells

o.n- over night

OVA-Ovalbumin

SA-Staphylococcus aureus

s.c.-subcutaneous

SEB-Staphylococcus aureus enterotoxin B

WT- Wild Type

\section{SUMMARY}

Background: Typical murine models of allergic inflammation are induced by the combination of ovalbumin and aluminum hydroxide. However, accumulating evidence indicates that, in models of asthma and atopic dermatitis, allergic inflammation can be generated in the absence of aluminum hydroxide. Moreover, coadministration of $S$. aureusenterotoxin B with ovalbumin can enhance inflammation.

Objective: The objective of this study was to establish a rapid and mast cell-dependent murine model of allergic inflammation by inducing allergic peritonitis using ovalbumin and $S$. aureusenterotoxin B.

Methods: Allergic peritonitis was induced in C57BL/6 mice by subcutaneous sensitization and intraperitoneal challenge with ovalbumin and $S$. aureus enterotoxin B. Disease characteristics were assessed by flow cytometry, ELISA, Trypan Blue exclusion and colorimetric assays.

Results: Time course of the allergic peritonitis revealed a peak of peritoneal inflammation $48 \mathrm{~h}$ after challenge, as assessed by total cells and eosinophil counts. Decrease of cell numbers started $96 \mathrm{~h}$ post challenge with complete clearance within 168h. Moreover, significantly higher levels of tryptase and increased vascular permeability were found $30 \mathrm{~min}$ following challenge. Allergic inflammation induction by ovalbumin and $S$. aureus enterotoxin B was impaired in mast cells deficient mice and partially restored by mice reconstitution with bone marrow derived mast cells, indicating the mast cell role in this model.

Conclusion: We present a novel model of allergic peritonitis that is mast cell-dependent, simple and robust. Moreover, the use of $S$. aureus enterotoxin B better resembles human allergic inflammation, which is known to be characterized by the colonization ofStaphylococcus aureus .

\section{INTRODUCTION}

Allergic diseases such as asthma, atopic dermatitis (AD), allergic rhinitis (AR) and food allergy affect $\sim 20 \%$ of the population worldwide (1). The most prominent cells involved in allergy are the mast cells (MCs), considered the primum movens of the reaction upon their activation by IgE-allergen, followed by the eosinophils (Eos), that heavily contribute to the inflammatory outcome. Moreover, their soluble and 
physical crosstalk that we identified and named the Allergic Effector Unit (AEU), is a key factor increasing the pro-inflammatory potential of the two cells and hence the allergic inflammation (AI) $(2,3)$. In addition, several reports have shown the nearly exclusive presence of $S$. aureus (SA) and its exotoxins, such as SA enterotoxin B (SEB) and others in asthma, AR, food allergy and AD (4-6). Interestingly, SA was found to directly bind and activate MCs (7) and Eos (8).

Until recently, the most common protocol used in the investigation of AI in mice involved the use of the antigen ovalbumin (OVA), together with aluminum hydroxide (Alum). However, it was shown that Alum can activate the innate immune response by activating the NLRP3 inflammasome through increasing uric acid levels (9). Furthermore, Alum was found to induce AI via activation of dendritic cells (DCs) independently of MCs (10). Indeed, it was shown that $24 \mathrm{~h}$ following intraperitoneal (i.p) injection, DCs and neutrophils were detected and increased during the inflammation in the peritoneal cavity, while MCs and macrophages, typical resident cells, decreased (10). In addition, Alum co-administration with an antigen was demonstrated not to be necessary for the induction of acute allergic airway inflammation (11) and of AD (12).

Noteworthy, BALB/C mice are often used in AI studies since they are prone to Th2 responses. Nevertheless, C57BL6 are employed more often because of the availability of transgenic and knockout (KO) strains based on their background $(13,14)$.

Therefore, our aim was to develop a mast cell-dependent, rapid and reproducible model of AI with the closest features to human Th2 allergic diseases in C57BL6 mice. Hence, we established an allergic peritonitis (AP) model by employing sensitization and challenge with OVA and SEB.

\section{MATERIALS AND METHODS}

\section{Mice}

Mast cell deficient KitW-sh/W-sh (Sash) mice (on a C57BL/6 background, purchased from Jackson Lab, Cambridge, MA, USA) were housed and bred in the pathogen free animal facilities of The Medical School of the Hebrew University of Jerusalem. C57BL/6 Wild Type (WT) mice were purchased from Harlan Laboratories Inc., Israel.

All murine experiments were approved by the Animal Experimentation Ethics Committee of the Hebrew University of Jerusalem and performed in accordance with the guidelines of the committee.

\section{Allergic Peritonitis (AP)}

8-9-week-old C57BL/6 (WT) females and/or males were sensitized on days -14 and -7 with subcutaneous

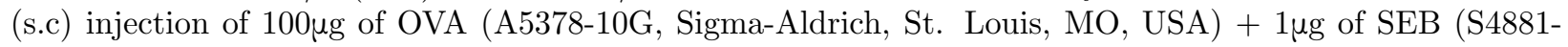

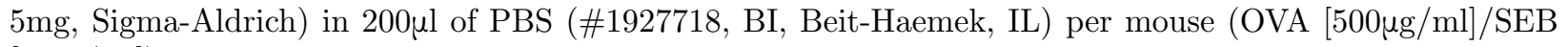
$[5 \mu \mathrm{g} / \mathrm{ml}])$ and challenged at day 0 with i.p injection of $10 \mu \mathrm{g}$ of OVA $+0.1 \mu \mathrm{g}$ SEB in $200 \mu \mathrm{l}$ of PBS per mouse $(\mathrm{OVA}[50 \mu \mathrm{g} / \mathrm{ml}] / \mathrm{SEB}[0.5 \mu \mathrm{g} / \mathrm{ml}])$ or PBS only. Mice were euthanized by $\mathrm{CO}_{2}$ inhalation on days 0 (0h), 2 (48h), $4(96 \mathrm{~h}), 7(168 \mathrm{~h})$ and $10(240 \mathrm{~h})$ and peritoneal lavage was performed with $3 \mathrm{ml}$ PBSX1+3\% FCS. Peritoneal cells were counted in hemocytometer by Trypan blue (\#03-102-1B, BI) staining. Peritoneal lavages were centrifuged at $150 \mathrm{~g}, 5 \mathrm{~min}, 4^{\circ} \mathrm{C}$ and supernatants were collected and stored or immediately used for further analysis. Cell pellets were resuspended in Flow Cytometry (FC) buffer $(0.5 \%$ BSA $+2 \%$ FCS in PBSX1) for FC analysis.

\section{FC analysis}

Cells were resuspended in a concentration of $3 X 10^{6}$ cells $/ \mathrm{ml}$ and $3 X 10^{5}$ cells $/ 100 \mu$ l were seeded per well of a 96 U-bottom plate. Cells were centrifuged $\left(150 \mathrm{~g} 5 \mathrm{~min}, 4^{\circ} \mathrm{C}\right)$, resuspended and incubated in $100 \mu \mathrm{l}$ blocking buffer (FC buffer $+5 \%$ goat serum) (15 min on ice). Thereafter, cells were centrifuged, resuspended and incubated with the following antibodies for 40 min: CD117 (c-Kit) monoclonal antibody (2B8), APC (\# 17-1171-82, ThermoFisher Scientific, Waltham, MA, USA), PE anti-mouse FceRI $\alpha$ antibody (\#134308, BioLegend, Inc., San Diego, CA, USA), PE Rat Anti-Mouse CD170 (Siglec-F) (\#552126,BD), APC anti-mouse CD193 (CCR3) antibody (144512, BioLegend, Inc.), PE anti-mouse F4/80 antibody (\#123109, BioLegend, 
Inc.), CD11b monoclonal antibody (M1/70), APC (\# 17-0112-82, ThermoFisher Scientific). All antibodies were matched with their corresponding isotype control: APC Rat IgG2b, $x$ Isotype Ctrl Antibody (\#400612, BioLegend, Inc.), Armenian Hamster IgG Isotype Control (eBio299Arm), PE (\# 12-4888-83, ThermoFisher Scientific.), PE Rat IgG2a, $x$ Isotype Ctrl Antibody (400508, BioLegend, Inc.), APC Rat IgG2a, $x$ Isotype Ctrl Antibody (400512, BioLegend, Inc.). After incubation, cells were washed twice with FC buffer, centrifuged and resuspended in 200 $\mu \mathrm{FC}$ buffer and acquired in a BD LSR II flow cytometer.

\section{sCD48 levels}

sCD48 levels were determined by using Mouse CD48 Quantification kit (Cat: K4-003-096) according to the manufacturer's instructions.

\section{Vascular Permeability}

The OVA/SEB AP model was induced and $1 \mathrm{~h}$ before the challenge, mice were injected intravenously (i.v.) with FITC-Dextran (\#46945-100mg, Sigma-Aldrich), 0.3mg / 150 $\mu$ in PBS per mice. Challenge was then performed, and mice were euthanized $30 \mathrm{~min}$ later and peritoneal lavage $(3 \mathrm{ml})$ was performed. Cells were separated from supernatants by centrifugation and supernatants were analyzed for FITC-Dextran levels in Cytation 3 Cell Imaging Multi-Mode Reader (BioTek Instruments, Inc; Winooski, VT, USA) using the following parameters: Excitation: $490 \mathrm{~nm}$, Emission: $520 \mathrm{~nm}$.

\section{Tryptase and cytokines levels in peritoneal lavage}

For assessing tryptase and cytokine levels in the peritoneal cavity, OVA/SEB AP-induced and challenged mice were euthanized $30 \mathrm{~min}$ or $48 \mathrm{~h}$ after challenge respectively and peritoneal lavages $(1 \mathrm{ml})$ were collected. Cells were separated from supernatants by centrifugation and supernatants were cleared by any remaining cell by a second centrifugation step. Tryptase levels were assayed as described before (15). Cytokines levels were determined by using IL-4 (900-K49; PeproTech, Rehovot, IL), INFg (900-K98; PeproTech) and IL-17A (900-K392; PeproTech) ELISA kits according to the manufacturer's instructions.

\section{OVA Specific IgE levels}

OVA specific IgE levels were determined by using LEGEND MAX ${ }^{\mathrm{TM}}$ Mouse OVA specific IgE ELISA kit (Cat: 4398071) according to the manufacturer's instructions.

\section{BMMCs sensitization with serum from immune OVA/SEB mice and activation with either OVA or SEB}

BMMCs were prepared as previously described (16). Thereafter, BMMCs were sensitized o.n with 1:5 diluted serum harvested from OVA/SEB challenged mice $48 \mathrm{~h}$ after the challenge. The day after sensitized BMMCs were washed and resuspended in BMMCs culture medium and activated for $24 \mathrm{~h}$ at $37^{\circ} \mathrm{C}$ with either $0.5 \mu \mathrm{g} / \mathrm{ml}$ OVA or $10 \mu \mathrm{g} / \mathrm{ml} \mathrm{SEB}$. After incubation supernatants were collected and analyzed for TNF $\alpha$ levels.

\section{Sash mice intraperitoneal reconstitution with bone marrow derived MCs (BMMCs)}

OVA/SEB AP model was induced in 6-12-week-old female Sash mice as described above. Twenty-four hours before challenge with OVA/SEB, mice were reconstituted i.p with $2 \times 10^{6}$ BMMCs/200 $\mu$ l PBS obtained from $\mathrm{C} 57 \mathrm{BL} / 6$. Mice were euthanized $48 \mathrm{~h}$ after challenge and peritoneal lavages $(3 \mathrm{ml})$ were processed as described above.

\section{Statistical analysis}

Data are expressed as mean \pm SEM. Statistical comparisons between experimental groups were performed using one-way ANOVA and post-hoc Tukey or Dunnett's multiple comparison tests. For less than three experimental groups, Student's unpaired two-tailed $t$-test was employed. Data were analyzed with Microsoft Excel (Microsoft, Redmond, Washington, USA) and Prism 6.0 (GraphPad Software, San Diego, California). A ' $p$ ' value of less than 0.05 was considered statistically significant for all analyses.

\section{RESULTS}




\section{OVA/SEB allergic peritonitis: cellular and soluble parameters.}

AP was induced in female C57BL/6 mice by OVA/SEB s.c sensitization (day 0 and 7 ) and challenge by i.p injection with lower concentrations of OVA/SEB on day 14 (Fig. 1A). As shown in Fig. 1B, in this model the peak of inflammation, as detected by the increase of total cells and Eos (CD170+/CD193+) numbers, was found $48 \mathrm{~h}$ following challenge. Total cells and Eos numbers started to decrease $96 \mathrm{~h}$ after challenge, returning to baseline levels (time point 0h) within 168h. MCs (CD117+/FCERI+) numbers did not display significant fluctuations with a trend of increase $48 \mathrm{~h}$ after challenge (Fig. 1B). Noteworthy, macrophages $(\mathrm{F} 4 / 80+/ \mathrm{CD} 11 \mathrm{~b}+)$ numbers increased significantly already at $48 \mathrm{~h}$, reaching a peak at $168 \mathrm{~h}$ and their number remained significantly higher than time point $0 \mathrm{~h}$ until 240h (Fig. 1B). Additionally, macrophages, as assessed at 48h, showed increased mRNA expression levels of the M1 marker iNOS (17), indicating a pro-inflammatory phenotype at this time point (S1). Moreover, sCD48 levels peaked 48h after challenged and returned to baseline levels at 168h (Fig. 1C). Noteworthy, C57BL/6 males showed a similar inflammation pattern, albeit less strong than in female mice (S2).

\section{OVA/SEB allergic peritonitis induces a mixed inflammation response.}

To further characterize our model, we analyzed peritoneal levels of IL-4, INFg and IL-17A, cytokine prototypes from Th1, Th2 and Th17 immune responses. Only IL-4 levels were significantly increased 48h following challenge (Fig. 2A), while INFg and IL-17A levels increased but not significantly (Fig. 2B-C). These results indicate that the OVA/SEB AP might induce a mixed inflammatory response shifted towards Th2 features.

\section{OVA/SEB allergic peritonitis induces production of OVA and SEB specific IgE.}

Next, we evaluated levels of OVA specific IgE in the serum of AP induced WT mice 48h after challenge. We found these antibodies to be increased, although not significantly, in WT OVA/SEB challenged mice in comparison to PBS challenged (Fig. 3A). In order to check OVA specific IgE together with SEB specific IgE, we analyzed WT BMMCs sensitized with serum retrieved from OVA/SEB challenged mice and activated them with either OVA or SEB. Our results show that, under these conditions, BMMCs were specifically activated by either OVA (significantly) or SEB (not significantly) to release TNF $\alpha$. (Fig. 3B). These data reconfirm that OVA/SEB mice produce IgE specific for both the allergens.

\section{OVA/SEB allergic peritonitis is a MC dependent model.}

Since one of our main aims was to establish a MC dependent model, we examined, 30 min post challenge in the OVA/SEB AP, tryptase and TNF $\alpha$ levels in the peritoneal lavage as markers of MCs degranulation. Both these preformed mediators were found to be significantly increased in the OVA/SEB-challenged mice in comparison to PBS-challenged ones (Fig. 4A-B). Likewise, vascular permeability in the peritoneum was significantly increased at the 30 min time point, further bolstering the $\mathrm{MC}$ role in this model (Fig. 4C).

Moreover, we induced the OVA/SEB AP model in MC deficient Sash mice. Forty-eight hours post challenge total cell infiltration into the peritoneal cavity was increased both in AP induced Sash mice and WT mice, although Sash mice presented slightly lower cell infiltration than WT ones (1.4- and 1.8-fold increase, respectively) (Fig. 5A). However, Eos numbers in the peritoneal cavity were significantly reduced in Sash OVA/SEB challenged mice in comparison to the WT group (Fig 5A). sCD48 levels in the peritoneal cavity were significantly augmented in the WT OVA/SEB group but not significantly in the Sash OVA/SEB group (Fig. 5B). Noteworthy, macrophages numbers showed a small albeit not significant increase in OVA/SEB challenged WT mice but no difference in the corresponding group of Sash mice (Fig. 5A). It is important to mention that in the OVA/SEB Sash mice group, two mice were found to have MCs in the peritoneal cavity and developed inflammation as WT mice. Therefore, they were excluded from the experiment (data not shown). To further dissect the role of MCs in our peritonitis model, Sash mice were reconstituted with BMMCs in the peritoneum in an "overshoot fashion" (2X106 cells) 24 hours before challenge (S3). Fortyeight hours post challenge, when MC numbers were found to be ${ }^{\sim} 10^{6}$ (Fig. 6A), inflammation was partially restored, as demonstrated by increments in total cell and in Eos numbers in comparison to non-reconstituted Sash mice (Fig 6A-B). 


\section{DISCUSSION}

In this paper we propose a new murine AP model induced by two antigens, OVA and SEB, for studying MCs dependent AI. OVA, a typical experimentally used antigen and allergen was administered together with SEB, that by itself is a superantigen, used as adjuvant. The SEB adjuvant-like activity was investigated before in an asthma model (18). It was shown that repeated sensitization with a combination of SEB together with OVA increased total cells and Eos number in bronchoalveolar lavage compared to sensitization with either OVA or SEB alone (18). Considering this data, we sought to use SEB to avoid the MCs independent inflammation induction by Alum (10). Moreover, we aimed to use a model that would include the typical S.aureus / exotoxins co-existence with the allergen taking place in allergy diseased patients. In our model we observed an inflammation pattern similar to what was previously reported for OVA/Alum AP (19). A difference was found though in the timing of the peak of inflammation that occurred in the OVA/Alum model at 72h- 96h after challenge in BALB/C mice $(19,20)$ and also in C57BL/6 (unpublished data). In the present OVA/SEB model the peak of inflammation is $48 \mathrm{~h}$ after challenge as indicated by the increase of Eos numbers a main characteristic of AI. This is in accordance with the results of Zuany-Amorim C et al, showing that OVA/Alum AP induction in BALB/C mice displayed a peak of inflammation at 48h (21). An additional explanation to this early peak could be provided by the superantigen role of SEB, as we previously found that SEB-induced peritonitis presented with increased total cell and Eos numbers $48 \mathrm{~h}$ after challenge (22). In our new model we also detected an increase in macrophages infiltration. This is not surprising, since it was previously reported that both SEB and OVA, respectively, increased macrophages numbers on the site of inflammation $(23,24)$. As expected, Eos and consequently total cell numbers decreased in the course of AP, while macrophages remained stable from $48 \mathrm{~h}$ after challenge. This might be the effect of the contribution of macrophages in both inflammation and resolution $(25,26)$. Indeed, we observed that macrophages phenotype in our model shifted towards M2, as shown by Arg1 (17) expression increasing during the course of AP (Supplemental Appendix Figure 1B). To further emphasize the induction of AI by using OVA/SEB AP, we analyzed SCD48 levels in peritoneal lavage at the detected peak of inflammation. It was previously published by our group that sCD48 levels correlate with Eos numbers in SEB-induced peritonitis (22). Furthermore, sCD48 levels in serum of mild asthma patients were significantly higher than in the control group and correlated with the Eos numbers (27). Consistently, in this model we found high levels of sCD48 in the peritoneal lavage at $48 \mathrm{~h}$ that returned to basal levels $168 \mathrm{~h}$ after challenge.

Many allergic diseases are characterized by the involvement of a mixed Th2 and Th1 (28-31) response and, as demonstrated by more recent evidence, of a Th17 one (32). For example, an AD murine model induced by OVA/SEB displayed increased mRNA levels of the Th2 cytokines IL-4 and IL-13, together with the Th1-related cytokines INFg and 12p40 (12). On the other hand, Bui et al. showed, in a murine OVA/Aluminduced asthma model, induction of IL-4 but not INFg release in mice bronchoalveolar lavage fluid (33). In addition, Bui et al. also showed an increase in the release of the Th17-related cytokine IL-17A (33). Significant levels of IL-17A, both at mRNA and protein levels were found in asthma patients (32). IL-17A was detected in the skin of OVA/SEB-induced AD mice (31). Correspondingly with the aforementioned evidence, our AP model showed significantly elevated levels of IL-4 simultaneously with a trend of increased levels of both IL-17A and INFg. These findings indicate that our model might induce a mixed Th1/Th2/Th17 response skewed towards Th2. After characterizing the inflammatory features of the OVA/SEB-induced AP, our next aim was to understand the involvement of MCs. We therefore evaluated MCs degranulation by measuring the levels of the MC preformed mediators, tryptase and $\mathrm{TNF} \alpha(34,35)$, in the peritoneal lavages. Indeed, we detected significantly higher levels of both mediators shortly after challenge (30 min), indicating that MCs are directly activated by OVA/SEB, possibly via IgE-dependent activation as we detected an increase in OVA specific IgE levels in the mice serum and SEB specific IgE, as shown by TNF $\alpha$ release from serum-sensitized BMMCs following activation with SEB. This is in accordance with previous reports showing that both OVA and SEB induce production of specific IgE during murine AI (12). In addition, it was shown that children suffering from AD presented with high levels of SEB specific IgE in their blood (36).

MC activation in vivo has been linked to increased vascular permeability (37). Indeed, this effect was found in our model as well, since $30 \mathrm{~min}$ after challenge we detected a significant increase in this parameter. To 
bolster the important role of MCs, we performed the OVA/SEB AP in MC-deficient (Sash) mice. Due to a mutation in the ckit regulatory element, Sash mice have MC deficiency in the peritoneal cavity and in additional sites at a young age, and in the skin as they age (38). Therefore, they have been extensively employed to assess the MC role in different pathological conditions. Sash mice sensitized and challenged with OVA/SEB presented attenuated inflammatory features such decreased recruitment of Eos and reduced release of sCD48. Reconstitution with BMMCs in the peritoneum in an overshoot protocol partly restored the inflammatory phenotype, as shown by the increase of peritoneal total cells and Eos numbers. Notably, BMMCs overshoot in the peritoneal cavity of PBS-challenged WT mice did not elicit Eos recruitment, demonstrating that the injected BMMCs did not induce inflammation by themselves (unpublished data).

In conclusion, the OVA/SEB-induced AP model is a Th2-skewed, MC-dependent AI model. This model is also closer to human pathology due to the presence of the SEB together with a potential allergen, such as OVA, that is relevant to human allergic diseases. Therefore, we suggest this model as an additional, useful tool to study AI.

ACKNOWLEDGEMENTS: H.P. planned and performed experiments, analyzed the data, prepared the figures, and wrote the manuscript. P.G.P. performed macrophage related experiments, assisted with in vivo experiments, analyzed the data and edited the manuscript. F.L.S. designed and supervised the study, analyzed the data, advised, reviewed and edited the manuscript.

The authors would like to thank Dr. Micha Ben-Zimra for helpful scientific discussions, Dr. Mansour Seaf for technical assistance and Ms. Alexandra Eliassaf (The Core Research Facility, The Faculty of Medicine, The Hebrew University of Jerusalem) who provided advice on the FC analysis and technical help.

The work was supported by grants from the Israel Science Foundation (ISF, 472/15), Vigevani Foundation, Rosetrees Trust (UK), Aimwell Trust (UK) to FLS. FLS is affiliated with the Adolph and Klara Brettler Center for Molecular Pharmacology and Therapeutics at the School of Pharmacy of The Hebrew University of Jerusalem.

Conflict of Interest: The authors report no conflicts of interest.

\section{REFERENCES}

1. Sánchez-Borges, M., B. L. Martin, A. M. Muraro, R. A. Wood, I. O. Agache, I. J. Ansotegui, T. B. Casale, T. A. Fleisher, P. W. Hellings, N. G. Papadopoulos, D. B. Peden, J. L. Sublett, S. A. Tilles, and L. Rosenwasser. 2018. The importance of allergic disease in public health: an iCAALL statement. World Allergy Organ. J. 11: 8.

2. Minai-Fleminger, Y., M. Elishmereni, F. Vita, M. R. Soranzo, D. Mankuta, G. Zabucchi, and F. LeviSchaffer. 2010. Ultrastructural evidence for human mast cell-eosinophil interactions in vitro. Cell Tissue Res. 341: 405-15.

3. Elishmereni, M., I. Bachelet, A. H. Nissim Ben-Efraim, D. Mankuta, and F. Levi-Schaffer. 2013. Interacting mast cells and eosinophils acquire an enhanced activation state in vitro. Allergy 68: 171-9.

4. Geoghegan, J. A., A. D. Irvine, and T. J. Foster. 2018. Staphylococcus aureus and Atopic Dermatitis: A Complex and Evolving Relationship. Trends Microbiol. 26: 484-497.

5. Tsilochristou, O., G. du Toit, P. H. Sayre, G. Roberts, K. Lawson, M. L. Sever, H. T. Bahnson, S. Radulovic, M. Basting, M. Plaut, G. Lack, and Immune Tolerance Network Learning Early About Peanut Allergy Study Team. 2019. Association of Staphylococcus aureus colonization with food allergy occurs independently of eczema severity. J. Allergy Clin. Immunol. 144: 494-503.

6. Flora, M., F. Perrotta, A. Nicolai, R. Maffucci, A. Pratillo, M. Mollica, A. Bianco, and C. Calabrese. 2019. Staphylococcus Aureus in chronic airway diseases: An overview. Respir. Med. 155: 66-71.

7. Rocha-de-Souza, C. M., B. Berent-Maoz, D. Mankuta, A. E. Moses, and F. Levi-Schaffer. 2008. Human mast cell activation by Staphylococcus aureus: interleukin- 8 and tumor necrosis factor alpha release and the 
role of Toll-like receptor 2 and CD48 molecules. Infect. Immun.76: 4489-97.

8. Minai-Fleminger, Y., R. S. Gangwar, H. Migalovich-Sheikhet, M. Seaf, V. Leibovici, N. Hollander, M. Feld, A. E. Moses, B. Homey, and F. Levi-Schaffer. 2014. The CD48 receptor mediates Staphylococcus aureus human and murine eosinophil activation. Clin. Exp. Allergy 44: 1335-46.

9. He, P., Y. Zou, and Z. Hu. 2015. Advances in aluminum hydroxide-based adjuvant research and its mechanism. Hum. Vaccin. Immunother. 11: 477-88.

10. Miki, H., C. Nakahashi-Oda, T. Sumida, and A. Shibuya. 2015. Involvement of CD300a Phosphatidylserine Immunoreceptor in Aluminum Salt Adjuvant-Induced Th2 Responses. J. Immunol. 194: 5069-76.

11. Conrad, M. L., A. O. Yildirim, S. S. Sonar, A. Kiliç, S. Sudowe, M. Lunow, R. Teich, H. Renz, and H. Garn. 2009. Comparison of adjuvant and adjuvant-free murine experimental asthma models. Clin. Exp. Allergy 39: $1246-54$.

12. Savinko, T., A. Lauerma, S. Lehtimäki, M. Gombert, M.-L. Majuri, N. Fyhrquist-Vanni, M.-C. DieuNosjean, L. Kemeny, H. Wolff, B. Homey, and H. Alenius. 2005. Topical superantigen exposure induces epidermal accumulation of CD8+ T cells, a mixed Th1/Th2-type dermatitis and vigorous production of $\operatorname{IgE}$ antibodies in the murine model of atopic dermatitis. J. Immunol. 175: 8320-6.

13. Gueders, M. M., G. Paulissen, C. Crahay, F. Quesada-Calvo, J. Hacha, C. Van Hove, K. Tournoy, R. Louis, J.-M. Foidart, A. Noël, and D. D. Cataldo. 2009. Mouse models of asthma: a comparison between C57BL/6 and BALB/c strains regarding bronchial responsiveness, inflammation, and cytokine production. Inflamm. Res. 58: 845-54.

14. Bryant, C. D. 2011. The blessings and curses of C57BL/6 substrains in mouse genetic studies. Ann. N. Y. Acad. Sci. 1245: 31-3.

15. Bachelet, I., A. Munitz, and F. Levi-Schaffer. 2005. Tryptase as an inflammatory marker in allergic disease and asthma. Expert Rev. Clin. Immunol. 1: 63-73.

16. Gangwar, R. S., and F. Levi-Schaffer. 2014. Eosinophils interaction with mast cells: the allergic effector unit. Methods Mol. Biol.1178: 231-49.

17. Orecchioni, M., Y. Ghosheh, A. B. Pramod, and K. Ley. 2019. Macrophage Polarization: Different Gene Signatures in M1(LPS+) vs. Classically and M2(LPS-) vs. Alternatively Activated Macrophages.Front. Immunol. 10: 1084.

18. Krysko, O., T. Maes, M. Plantinga, G. Holtappels, R. Imiru, P. Vandenabeele, G. Joos, D. V Krysko, and C. Bachert. 2013. The adjuvant-like activity of staphylococcal enterotoxin B in a murine asthma model is independent of IL-1R signaling. Allergy 68: 446-53.

19. Karra, L., R. Singh Gangwar, R. Shamri, P. G. Puzzovio, S. Cohen-Mor, B. D. Levy, and F. Levi-Schaffer. 2018. Leukocyte CD300a Contributes to the Resolution of Murine Allergic Inflammation. J. Immunol. 201: 2998-3005.

20. Temkin, V., D. Pickholtz, and F. Levi-Schaffer. 2003. Tumor necrosis factors in a murine model of allergic peritonitis: effects on eosinophil accumulation and inflammatory mediators' release. Cytokine 24: 74-80.

21. Zuany-Amorim, C., D. Leduc, B. B. Vargaftig, and M. Pretolani. 1993. Characterization and pharmacological modulation of antigen-induced peritonitis in actively sensitized mice. Br. J. Pharmacol. 110: 917-24.

22. Gangwar, R. S., and F. Levi-Schaffer. 2016. sCD48 is anti-inflammatory in Staphylococcus aureus Enterotoxin B-induced eosinophilic inflammation. Allergy 71: 829-39.

23. Wang, X., H. Zhao, C. Ma, L. Lv, J. Feng, and S. Han. 2018. Gallic acid attenuates allergic airway inflammation via suppressed interleukin-33 and group 2 innate lymphoid cells in ovalbumin-induced asthma in mice. Int. Forum Allergy Rhinol. 8: 1284-1290. 
24. Herz, U., R. Rückert, K. Wollenhaupt, T. Tschernig, U. Neuhaus-Steinmetz, R. Pabst, and H. Renz. 1999. Airway exposure to bacterial superantigen (SEB) induces lymphocyte-dependent airway inflammation associated with increased airway responsiveness-a model for non-allergic asthma. Eur. J. Immunol. 29: $1021-31$.

25. Watanabe, S., M. Alexander, A. V. Misharin, and G. R. S. Budinger. 2019. The role of macrophages in the resolution of inflammation.J. Clin. Invest. 129: 2619-2628.

26. Wynn, T. A., A. Chawla, and J. W. Pollard. 2013. Macrophage biology in development, homeostasis and disease. Nature 496: 445-55.

27. Gangwar, R. S., Y. Minai-Fleminger, M. Seaf, A. Gutgold, A. Shikotra, C. Barber, A. Chauhan, S. Holgate, P. Bradding, P. Howarth, R. Eliashar, N. Berkman, and F. Levi-Schaffer. 2017. CD48 on blood leukocytes and in serum of asthma patients varies with severity.Allergy 72: 888-895.

28. Nayak, A. P., T. L. Croston, A. R. Lemons, W. T. Goldsmith, N. B. Marshall, M. L. Kashon, D. R. Germolec, D. H. Beezhold, and B. J. Green. 2018. Aspergillus fumigatus viability drives allergic responses to inhaled conidia. Ann. Allergy. Asthma Immunol. 121: 200-210.e2.

29. Smart, J. M., and A. S. Kemp. 2002. Increased Th1 and Th2 allergen-induced cytokine responses in children with atopic disease. Clin. Exp. Allergy 32: 796-802.

30. Minang, J. T., I. Areström, M. Troye-Blomberg, L. Lundeberg, and N. Ahlborg. 2006. Nickel, cobalt, chromium, palladium and gold induce a mixed Th1- and Th2-type cytokine response in vitro in subjects with contact allergy to the respective metals. Clin. Exp. Immunol.146: 417-26.

31. Karra, L., R. S. Gangwar, P. G. Puzzovio, N. Fyhrquist, Y. Minai-Fleminger, N. Landolina, H.-U. Simon, H. Alenius, V. Leibovici, D. Simon, and F. Levi-Schaffer. 2019. CD300a expression is modulated in atopic dermatitis and could influence the inflammatory response.Allergy 74: 1377-1380.

32. Gupta, R. K., K. Gupta, and P. D. Dwivedi. 2017. Pathophysiology of IL-33 and IL-17 in allergic disorders. Cytokine Growth Factor Rev. 38: 22-36.

33. Bui, T. T., C. H. Piao, C. H. Song, H. S. Shin, D.-H. Shon, and O. H. Chai. 2017. Piper nigrum extract ameliorated allergic inflammation through inhibiting Th2/Th17 responses and mast cells activation. Cell. Immunol. 322: 64-73.

34. Robida, P. A., P. G. Puzzovio, H. Pahima, F. Levi-Schaffer, and B. S. Bochner. 2018. Human eosinophils and mast cells: Birds of a feather flock together. Immunol. Rev. 282: 151-167.

35. Olszewski, M. B., A. J. Groot, J. Dastych, and E. F. Knol. 2007. TNF trafficking to human mast cell granules: mature chain-dependent endocytosis. J. Immunol. 178: 5701-9.

36. Bunikowski, R., M. Mielke, H. Skarabis, U. Herz, R. L. Bergmann, U. Wahn, and H. Renz. 1999. Prevalence and role of serum IgE antibodies to the StaphylococcuS aureus-derived superantigens SEA and SEB in children with atopic dermatitis. J. Allergy Clin. Immunol. 103: 119-124.

37. Kunder, C. A., A. L. St John, and S. N. Abraham. 2011. Mast cell modulation of the vascular and lymphatic endothelium. Blood 118: 5383-93.

38. Grimbaldeston, M. A., C.-C. Chen, A. M. Piliponsky, M. Tsai, S.-Y. Tam, and S. J. Galli. 2005. Mast cell-deficient $\mathrm{W}$-sash c-kit mutant Kit W-sh/W-sh mice as a model for investigating mast cell biology in vivo.Am. J. Pathol. 167: 835-48.

\section{FIGURE LEGENDS}

\section{Figure 1: Time course of inflammation in the OVA/SEB AP.}

(A ) OVA/SEB AP was carried out in 8-9 weeks old C57BL/6 WT female mice. Inflammation was measured, at different time points, by (B ) peritoneal total cells (Trypan blue), Eos, MCs and macrophages (FC 
Analysis) cell numbers, and $(\mathbf{C})$ sCD48 levels (ELISA). Data are the mean $+/-$ SEM, $\mathrm{n}=4-5$ mice per group, ${ }^{*} \mathrm{p}<0.05,{ }^{* *} \mathrm{p}<0.01,{ }^{* * *} \mathrm{p}<0.001$.

Figure 2: Cytokine levels from total cells in the peritoneal lavage of C57BL/6 mice following AP model.

(A ) IL-4, (B ) IL-17A and (C ) INF $\gamma$ levels in the peritoneal lavage of PBS- or OVA/SEB- challenged mice $48 \mathrm{~h}$ after challenge. Data are the mean $+/-\mathrm{SEM}, \mathrm{n}=4$ mice/group, ${ }^{*} \mathrm{p}<0.05$.

Figure 3: OVA and SEB specific IgE production in OVA/SEB AP

(A ) OVA specific IgE levels in the sera of WT OVA/SEB- or PBS-challenged mice 48h following challenge. (B ) TNF $\alpha$ release from serum-sensitized, OVA or SEB activated WT BMMCs. Data are the mean +/SEM, $\mathrm{n}=3 ;{ }^{*} \mathrm{p}<0.05$.

Figure 4: Vascular permeability and soluble mediators release in the peritoneal lavage of C57BL/6 mice following AP model.

(A ) Tryptase, (B ) TNF $\alpha$ levels in peritoneal lavage and (C) vascular permeability in the peritoneal cavity of C57BL/6 mice 30min following challenge as measured by FITC-Dextran levels in peritoneal lavage. Data are the mean $+/-$ SEM, $\mathrm{n}=5-7$ mice/group; ${ }^{*} \mathrm{p}<0.05,{ }^{*} \mathrm{p}<0.01$.

Figure 5: OVA/SEB AP model in MC-deficient mice.

OVA/SEB AP was carried out in 8-9 weeks old C57BL WT and Sash female mice. Inflammation was measured at $48 \mathrm{~h}$ time point by (A ) peritoneal total cells (Trypan blue), Eos, MCs and macrophages (FACS Analysis) cell numbers, $\mathrm{n}=6-8$ mice per group (B ) sCD48 levels (ELISA) $\mathrm{n}=3-5$ mice per group. Data are the mean $+/-$ SEM, ${ }^{*} \mathrm{p}<0.05,{ }^{* *} \mathrm{p}<0.01,{ }^{* * *} \mathrm{p}<0.001$.

Figure 6: OVA/SEB AP inflammation after BMMCs reconstitution (Overshoot) in MC deficient mice.

(A ) Total cell and (B ) Eosinophils in peritoneum lavage of WT mice or Sash mice, with or without reconstitution of WT BMMCs, 48h after challenge. Data are the mean +/- SEM, n=4-6 mice/group, ${ }^{*} \mathrm{p}<0.05$.

\section{Figure 1:}

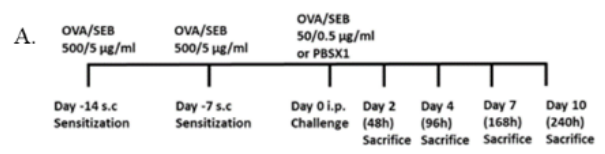

B. Total cell number
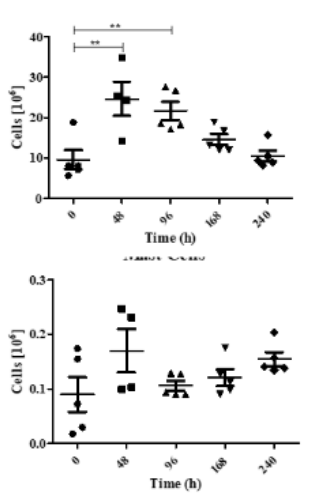
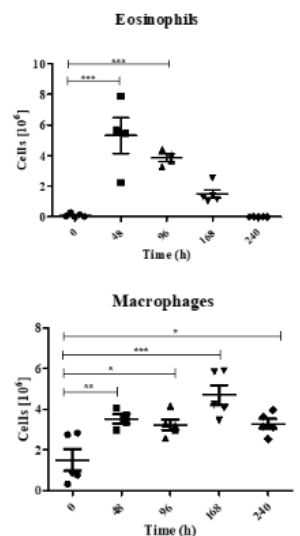

C.

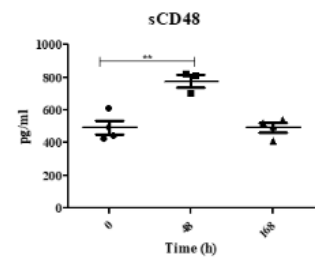

Figure 2: 

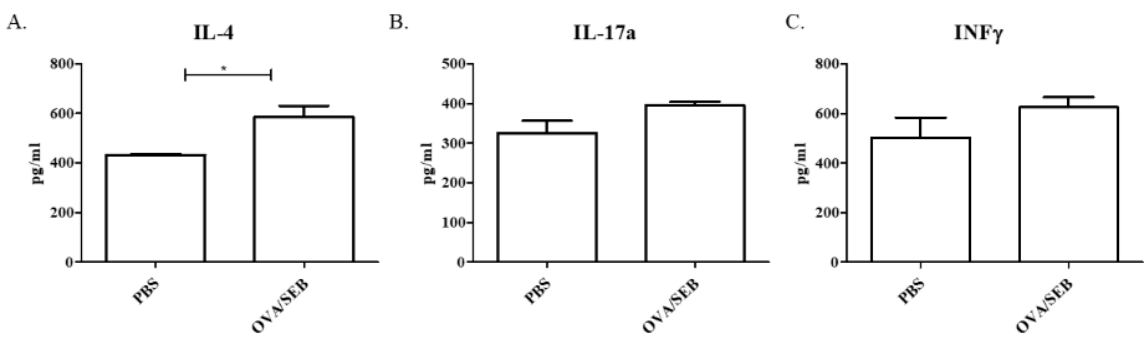

Figure 3:

A.

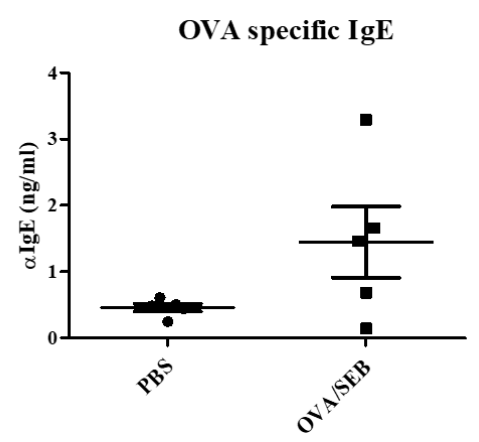

Figure 4:
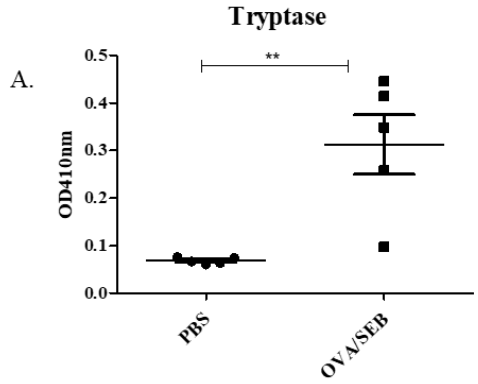

B.

TNF $\alpha$ levels

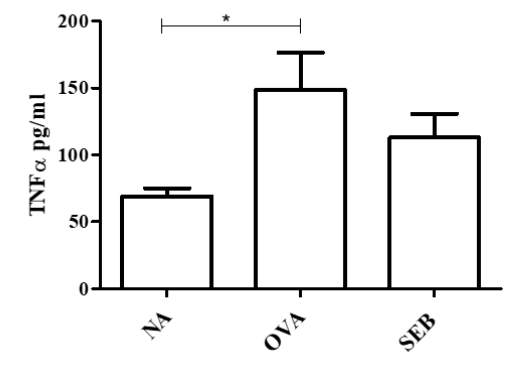

C.

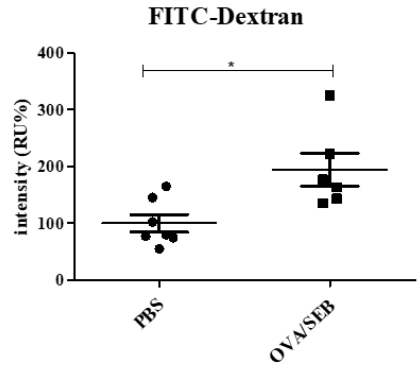

Figure 5: 

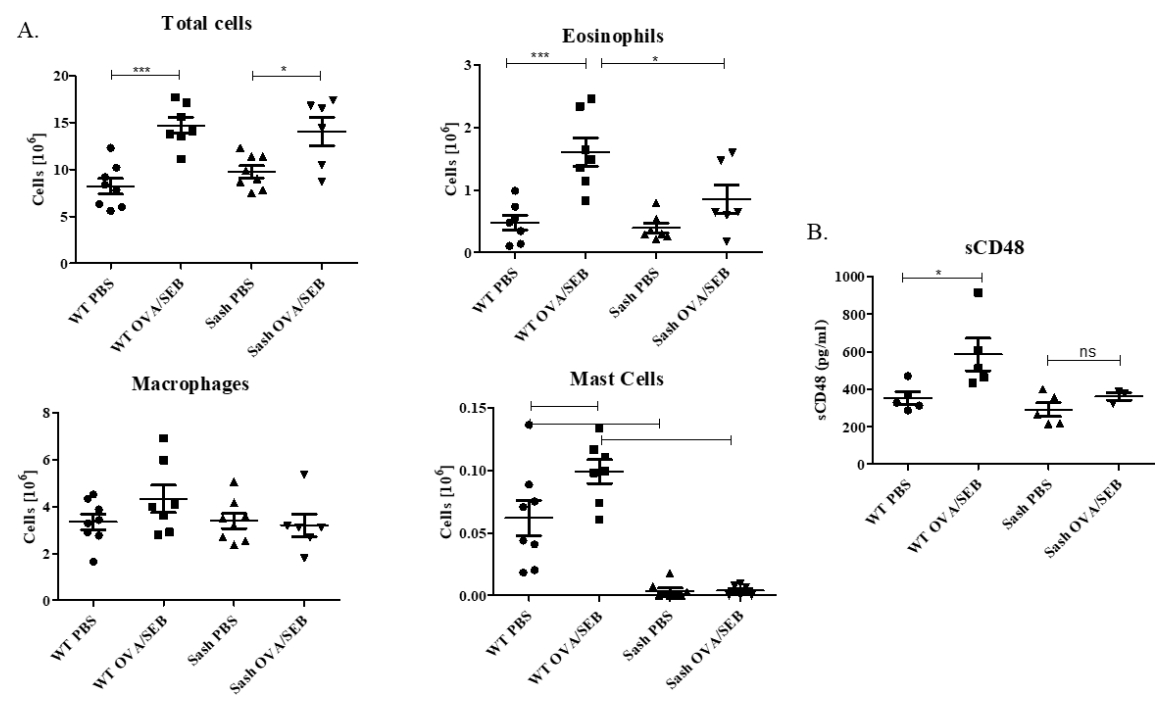

Figure 6:

Total cell

A.

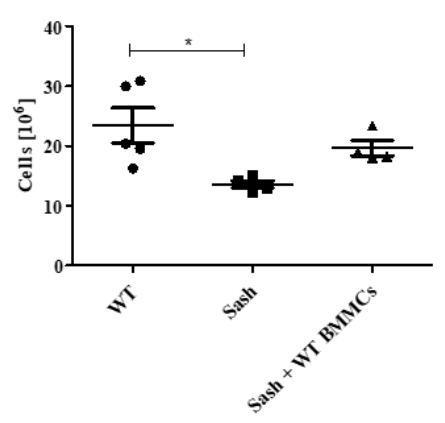

B.

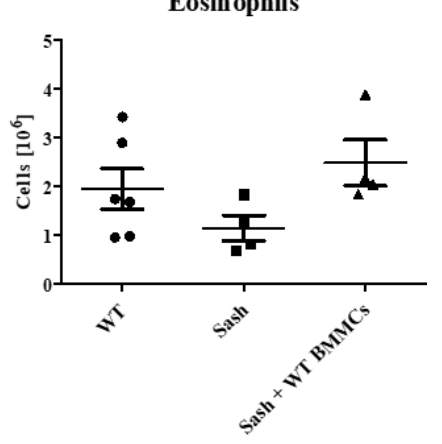

